\title{
Histoire et identite : l'exemple du Crédit Agricole
}

\section{Christian Bosséno}

\section{OpenEdition}

Journals

Édition électronique

URL : http://journals.openedition.org/communicationorganisation/1783

DOI : 10.4000/communicationorganisation. 1783

ISSN : 1775-3546

\section{Éditeur}

Presses universitaires de Bordeaux

\section{Édition imprimée}

Date de publication : 1 mai 1995

ISSN : 1168-5549

\section{Référence électronique}

Christian Bosséno, «Histoire et identite : l'exemple du Crédit Agricole », Communication et organisation [En ligne], 7 | 1995, mis en ligne le 26 mars 2012, consulté le 19 avril 2019. URL : http:// journals.openedition.org/communicationorganisation/1783; DOI : 10.4000/ communicationorganisation. 1783

Ce document a été généré automatiquement le 19 avril 2019

(c) Presses universitaires de Bordeaux 


\title{
Histoire et identite : l'exemple du Crédit Agricole
}

\author{
Christian Bosséno
}

1 "Le présent sans le passé n'a pas d'avenir » constate Fernand Braudel. De fait, une entreprise puise sa force, les principes de son action, son identité, dans sa mémoire. A fortiori quand elle a le privilège, comme c'est le cas pour le Crédit Agricole, d'être fondée sur une histoire originale et des valeurs fortes.

2 Or, privilégiant souvent le court terme, l'entreprise française est quelquefois amnésique, comme si, confusément, l'évocation du passé devait être nécessairement source de nostalgie, signifier le strict respect de la tradition, des héritages, des positions acquises, bref constituer autant de freins aux nécessaires mutations.

3 Cet état d'esprit explique sans doute que les entreprises françaises aient, contrairement aux anglosaxonnes et aux allemandes, souvent tardé à s'appuyer sur leur histoire pour définir leur stratégie et construire leur développement. Cette carence, entretenue par l'euphorie de l'expansion des « 30 glorieuses", où la croissance pour la croissance apparaissait comme la seule justification de l'entreprise, s'est révélée, à l'épreuve de la crise, comme réductrice.

4 Car, bien au contraire, en renforçant l'identité de l'entreprise, l'histoire peut efficacement en aider le management. Chacune, en effet, génère une culture propre fondée sur des valeurs partagées par l'ensemble de son personnel et destinées à s'enrichir.

5 Renforcer l'identité de l'entreprise, connaître et cultiver son histoire, c'est mettre en évidence sa culture et ses valeurs, tisser des liens solides entre ses agents.

6 À l'évidence, ce patrimoine se trouve réparti dans toute l'entreprise et entre tous ses acteurs. Ainsi au Crédit Agricole dans chacune de ses composantes (Caisses locales, Caisses régionales et leurs agences, Caisse nationale et ses filiales, en France et à l'étranger). À quoi il faut bien entendu ajouter les retraités de l'institution, qui peuvent enrichir la mémoire de l'entreprise par leurs documents personnels ou leurs témoignages. C'est pourquoi l'histoire d'entreprise y est l'affaire de tous, élus et salariés, pour alimenter le fonds de mémoire du Groupe. 
7 Ces gisements étant restés très longtemps en jachère, la tâche la plus urgente était, lors de la création de la Mission historique de la CNCA en juin 1990, de prendre des mesures conservatoires pour constituer un fonds de mémoire, de recueillir les témoignages, d'organiser et de gérer des archives historiques. Ce travail considérable est aujourd'hui loin d'être terminé. C'est donc la jeune expérience de la Mission Archives/Histoire, que je dirige, que je me propose de décrire ici, en rappelant parallèlement quelques traits essentiels de l'identité du Groupe Crédit Agricole, qui constituent le socle de ses avancées futures.

\section{L'histoire, un métier neuf au Crédit Agricole}

8 Le Crédit Agricole vient de célébrer le centenaire de son acte fondateur: la loi du 5 novembre 1894, votée à l'unanimité, donnant un statut aux "sociétés de Crédit Agricole». L'histoire de ce Groupe, fondé sur des valeurs originales comme l'esprit mutualiste, la priorité du service, l'engagement des hommes et la solidarité, constitue aujourd'hui pour le Crédit Agricole une source d'inspiration d'autant plus essentielle qu'après avoir été longtemps spécialisé dans le financement de l'agriculture, il est parvenu au terme de l'extension de ses champs de compétences, diversifiant ses opérations et ses métiers et exerçant désormais pleinement la fonction de banquier.

Ce n'est pourtant qu'en 1990 qu'une Mission historique a été instituée à la Caisse nationale de Crédit Agricole, à l'initiative du président Yves Barsalou et du directeur général Philippe Jaffré1. Son objectif était triple. Il était d'abord de sauvegarder et d'organiser les archives historiques. Il était ensuite d'utiliser ces matériaux pour acculturer l'ensemble du personnel, notamment les nouveaux recrutés, mais aussi pour rédiger à l'intention du Président et du Directeur général de la CNCA, en fonction de leurs déplacements ou des problèmes d'actualité, des notes historiques ponctuelles. Il était enfin de mettre en valeur le patrimoine du Groupe par l'organisation d'expositions, la publication d'articles (dans la revue d'entreprise ou des supports extérieurs) et d'ouvrages historiques, d'assister les Caisses régionales et les filiales pour toutes manifestations ou travaux concernant l'histoire.

$10 \mathrm{Au}$ vrai, et même si cette initiative est relativement récente, le Crédit Agricole s'est engagé parmi les tout premiers dans le secteur bancaire sur cette voie, précédé seulement par la Caisse des dépôts et consignations. Après lui, d'autres établissements bancaires (Paribas, Crédit lyonnais, Banque de France) ont institué des unités d'inspiration voisine et ce mouvement devrait se généraliser tant est forte aujourd'hui la prise de conscience dans les banques de l'utilité d'une démarche historique.

\section{Premiers travaux et éléments d'une méthode}

11 Pour constituer les bases de ce fonds d'archives nécessaires à toute production, la première tâche de la Mission a été de rassembler et de recenser les matériaux qui, dispersés tant à la Caisse nationale que dans les Caisses régionales, pouvaient encore exister. Cette préoccupation était généralement nouvelle. En effet, le Crédit Agricole ayant connu depuis les années cinquante une phase d'expansion sans précédent dans le secteur bancaire, qui a entraîné au niveau national et régional des déménagements de sièges sociaux, la constitution et la conservation d'archives historiques avait alors le plus souvent été négligée dans l'euphorie de la « conquête ». 

chacune des Caisses régionales pour tenter une première estimation du patrimoine historique du groupe, afin de localiser les "gisements » internes et poser les bases d'un inventaire national. Dans le même temps était constitué un réseau de correspondants, chaque Caisse régionale désignant un cadre chargé d'entretenir des relations suivies avec la Mission. Très vite s'établirent des relations dans les deux sens, les Caisses régionales alimentant par leurs recherches le patrimoine commun, la Mission historique apportant aux Caisses régionales son concours et son expertise pour leurs recherches et leurs travaux historiques (manifestations, publication de monographies). Cette action a reçu l'appui constant et personnel du Président et du Directeur général de la CNCA, cette adhésion étant un point capital pour le développement et le succès de la Mission. Des relations étroites et permanentes ont également été nouées avec les associations de retraités (Caisse nationale et cadres de direction des Caisses régionales).

D'autre part une prospection de toutes les sources utiles à la constitution d'un fonds historique a été entreprise: archives nationales et départementales, cinémathèques régionales, librairies spécialisées, clubs de collectionneurs, presse spécialisée pour toute consultation ou acquisition extérieure, etc...

Des outils de travail internes ont pu ainsi, et dans un délai très rapide, être constitués au bénéfice de tous (éléments méthodologiques, catalogue d'affiches édité et enrichi chaque année depuis 1992, catalogue des films et vidéos édité pour la première fois en 1994). Dans le même temps, quelques mois après la création de la Mission, une plaquette d'une quarantaine de pages était publiée, La Caisse nationale dans le Crédit Agricole, une histoire dans l'histoire ${ }^{2}$, et une première exposition sur l'histoire de la CNCA, organisée à SaintQuentin-en-Yvelines à l'occasion de la manifestation accompagnant la publication du projet d'entreprise de la Caisse nationale. Il s'agissait en effet de "produire " très rapidement, pour démontrer l'intérêt des recherches historiques, les légitimer auprès de tous et obtenir une adhésion de l'ensemble des salariés à ce nouveau chantier, dans un groupe caractérisé à la fois par son unité et sa décentralisation.

15 D'emblée, le caractère fédérateur du projet a pu ainsi susciter un véritable engouement. De plus, une réunion annuelle des correspondants de la Mission historique, ouverte par le Directeur général de la CNCA, permet, depuis 1992, de nourrir une réflexion d'ensemble, de coordonner les travaux, de mettre en commun des expériences et des recherches et de maintenir un contact entretenu en cours d'année par des échanges réciproques.

16 Au plan interne, la Mission est prestataire de services pour l'essentiel des directions et filiales de la Caisse nationale. C'est ainsi par exemple qu'elle ouvre régulièrement, sous l'égide de la direction des Ressources humaines, le cycle d'accueil des nouveaux salariés, qu'elle est intervenue à la succursale de Londres et qu'elle a participé à un séminaire destiné aux personnels des implantations du Crédit Agricole à l'étranger.

17 Cette démarche identitaire a été entreprise dans une période de mutation profonde $d u$ Crédit Agricole après le passage de la Caisse nationale du statut d'établissement public à celui de société anonyme (1988) et la perte du monopole des prêts bonifiés à l'agriculture (1990). Une période également marquée par la poursuite de la diversification des activités du Groupe avec la création de sociétés d'assurances complétant la gamme des services à la clientèle, et la multiplication des métiers très spécialisés nécessitant de nouveaux recrutements. Dans le même temps était amorcé le rapprochement des Caisses régionales, visant à constituer des unités de taille compatible avec le marché européen et induisant 
un redéploiement des effectifs. La taille grandissante du Crédit Agricole et l'éclatement géographique des salariés de la CNCA et de ses filiales, l'internationalisation des marchés financiers, mais aussi l'accélération du turn-over pouvaient amener sinon à s'interroger sur l'identité du Groupe, du moins à y réfléchir.

Ces mutations se sont accompagnées, dans le cadre d'un projet de Groupe, d'une redéfinition du rôle de chaque entité (Caisse nationale et ses filiales, Fédération nationale du Crédit Agricole, Caisses régionales) et de la détermination de nouveaux axes stratégiques.

Or, c'est précisément dans un tel contexte évolutif que l'appropriation de l'histoire et la prise en compte des valeurs fondatrices prennent tout leur sens, légitimant le labeur des aînés et ancrant chez les jeunes recrutés une connaissance de l'entreprise qui nourrit leur sentiment d'appartenance. C'est quand le modèle paraît se brouiller que l'histoire vient relativiser les changements, les expliquer, et, quelquefois, les dédramatiser en les inscrivant dans une perspective dynamique d'évolution ${ }^{3}$.

\section{Un siècle au présent : la célébration}

20 Dans ce contexte, la célébration du centenaire du Crédit Agricole a constitué un événement fédérateur. Sous la dénomination générique "Un siècle au présent », cette manifestation, d'abord nationale et qui a ensuite été déclinée dans les Caisses régionales, selon la sensibilité propre et l'histoire singulière de chacune, a incontestablement marqué un temps fort, à la fois de découverte et de réflexion, dans la vie du Groupe, une étape pour mieux considérer l'avenir.

21 Au plan national, en septembre 1994, une manifestation (rééditée au mois de décembre pour l'ensemble du personnel du groupe Caisse nationale) a réuni au Palais des Congrès à Paris des administrateurs et des cadres de Caisses régionales. Le programme, basé sur les matériaux réunis par la Mission historique et orchestré par les directions de la Communication de la CNCA et de la FNCA avec le concours des Caisses régionales, se composait :

22 - d'une reconstitution théâtrale de l'«acte fondateur », dont le texte avait été composé directement à partir des débats parlementaires et dont les protagonistes (interprétés par des acteurs professionnels) étaient Jules Méline, l'initiateur de la loi, Albert Viger, ministre de l'Agriculture, Jean Jaurès, auteur d'un projet concurrent, Clemenceau, etc. ;

23 - de la projection d'une série de vidéos résultant du montage de documents d'archives retrouvés et déroulant l'histoire du Crédit Agricole mise en perspective ;

24 - de tables rondes thématiques;

25 - de la projection d'un film du centenaire, mêlant documents d'archives et reconstitutions inspirées par l'histoire ;

26 - d'allocutions de personnalités et des dirigeants du Groupe.

27 Dans les semaines qui suivirent, chaque Caisse régionale, se basant sur ses recherches propres et des scénarios imaginés par elle, organisa à son tour une manifestation originale, pour laquelle elle a pu bénéficier de l'appui de la CNCA et de la FNCA. 


\section{Un siècle au présent : le livre}

28 intitulé, comme l'événement qu'il contribue à marquer, Un Siècle au Présent. Ce volume retrace les premières tentatives d'institution d'une banque de Crédit Agricole, puis raconte l'histoire du Crédit Agricole mutuel des origines à 1950 (un second tome traitant de la période plus contemporaine sera publié en 1995 à l'occasion de la Convention nationale du Crédit Agricole). Le principe qui a présidé à la rédaction de l'ouvrage a été de proposer un véritable travail historique répondant à des critères scientifiques, mais aussi ludiques en provoquant l'étonnement, voire le «dépaysement » du lecteur. L'ouvrage, abondamment illustré, propose plusieurs niveaux de lecture : contexte historique en bas des pages et dans un graphisme différent, encadrés et légendes constituant une autre dimension de lecture. Il est à noter que pour plus de $90 \%$, l'iconographie est le fruit de recherches de la Mission historique. Souvent inédite, elle a contribué à l'impact de l'ouvrage $e^{4}$.

La rédaction, elle aussi, résulte d'une démarche particulière dans la mesure où elle a été le fruit du travail d'écriture conjoint du responsable de la Mission historique (s'appuyant également sur des apports des correspondants en Caisses régionales) et d'un historien, André Gueslin, professeur à l'Université de Paris VII et auteur, en 1983, d'une thèse d'État sur l'histoire des crédits agricoles ainsi que de plusieurs ouvrages sur le même thème. La direction de la Communication a coordonné la révision éditoriale et a assuré la diffusion de l'ouvrage.

Cette confrontation d'approches différentes a permis la rédaction d'un livre original qui a également bénéficié, pour son élaboration, de l'examen critique d'un groupe de " sages » composé de cadres de direction retraités de la Fédération ou de la Caisse nationale, ayant pour certains exercé des fonctions de direction en Caisses régionales.

\section{Perspectives}

31 Si la célébration du centenaire du Crédit Agricole a constitué un moment très fort d'impulsion et d'émotion, elle ne marque pas bien évidemment le terme du rôle de la Mission historique, conçue comme une structure pérenne, associée au management de la Caisse nationale de Crédit Agricole.

Elle s'inscrit dans une succession de chantiers ouverts régulièrement: anniversaires de filiales, projets menés en collaboration avec les Caisses régionales pour leur propre production de nouveaux ouvrages. De la même façon, la Mission historique s'est ouverte sur l'extérieur, tant en nouant des relations avec les services historiques d'autres banques (notamment dans le cadre de European Association for Banking History - EABH), qu'en commençant d'accueillir les chercheurs et les universitaires à l'occasion de leurs travaux.

Mais le chantier qui exigera sans doute le plus de ce jeune service, riche déjà d'une activité de production importante et reconnu par l'ensemble des composantes du Groupe, sera l'organisation et la gestion des fonds d'archives déjà réunis, ainsi que la mise en place d'un système permettant de sélectionner dans les documents d'aujourd'hui ceux qui seront destinés à nourrir les archives historiques, tant au niveau de la CNCA que des Caisses régionales.

Communication et organisation, 7 | 1995 
Après l'euphorie des premières réalisations, c'est à ce chantier fondamental qu'il nous faut aujourd'hui œuvrer!

Quelques traits marquants de l'identité originale du Crédit Agricole

- Une appartenance mutualiste réaffirmée. Le Crédit Agricole a été fondé sur des valeurs mutualistes, mises en œuvre par les Caisses locales (1894) et régionales (1899), banques coopératives à capital variable. Il continue de les revendiquer aujourd'hui dans son projet de Groupe (1989).

- Le Crédit Agricole naît d'initiatives privées, mais reste longtemps appuyé par l'État. Au début, ce sont les fonctionnaires de la République (préfets, professeurs départementaux d'agriculture, instituteurs) qui le font connaître. Dans le même temps, pour pallier l'insuffisance des ressources collectées, des fonds publics sont mis à sa disposition (dotation et redevances de la Banque de France puis crédits budgétaires).

- Une construction pragmatique. Il se construit pragmatiquement et progressivement en 3 étapes, de la base au sommet : Caisses locales (1894), Caisses régionales (1899), Caisse nationale (1920).

- Une expansion sans précédent. Organisme peu connu en dehors du monde rural au sortir de la deuxième guerre mondiale, le Crédit Agricole ne "pèse » alors que $5 \%$ de l'ensemble des bilans bancaires. À partir des années cinquante, pour pouvoir, malgré la diminution des ressources d'État, pleinement jouer son rôle de financier de l'agriculture, dont les besoins considérables, il entreprend une vigoureuse action de développement de sa collecte de dépôts et d'épargne. Et c'est la surprise générale, quand, dans le classement des banques pour 1975, le " petit poucet de la finance » apparaît au 3e rang mondial ! Il se hissera même au premier rang en $1979 . .$.

- Une institution composite jusqu'en 1988. Pendant près de 70 ans, le Crédit Agricole est un mariage original entre deux échelons de droit privé, les Caisses locales et régionales (banques coopératives à capital variable), et un établissement public central, la CNCA. En 1988, la CNCA est «mutualisée », c'est-à-dire privatisée. Cette réforme institutionnelle émancipe le Crédit Agricole de la tutelle de l'état.

1 - Un groupe uni et décentralisé. Groupe décentralisé, caractérisé par l'autonomie de chaque Caisse régionale, le Crédit Agricole est aussi un groupe uni autour d'une Caisse nationale forte.

2 La Fédération nationale du Crédit Agricole, refondée en 1945 comme représentation des Caisses régionales, constitue un centre d'études et de réflexion.

- De l'établissement spécialisé à la banque universelle. Cantonné, à l'origine, au seul financement de l'agriculture, le Crédit Agricole entreprend, à partir de 1959, une « longue marche » vers la banque universelle, qui aboutira totalement en 1991.

En effet, le Crédit Agricole vit un paradoxe. Dès le début, il est habilité à recevoir des fonds de toute personne. En revanche, il ne peut prêter qu'à ses sociétaires, c'est-à-dire les agriculteurs et leurs groupements. Les besoins considérables du financement de la reconstruction et de la modernisation de l'agriculture qui s'expriment après la seconde guerre mondiale et la volonté de désengagement de l'État le contraignent à développer sa collecte dans le milieu rural, puis dans les villes. L'obligation de réciprocité (pouvoir prêter à ses déposants) le conduit à mener, de 1959 à 1991, une action opiniâtre auprès des pouvoirs publics pour obtenir l'extension progressive de ses compétences. 

l'engagement des Caisses régionales sur le terrain entrâne une progression sans équivalent de la collecte.

- La création des filiales. Une stratégie complémentaire, la création d'un réseau de filiales, notamment de filiales de financement, est amorcée dès 1961 pour repousser les frontières et conquérir de nouveaux territoires de compétence.

47 - La « démocratisation de la banque ». En des temps où les banques pratiquent une stricte sélectivité, le Crédit Agricole saisit toutes les opportunités d'élargir sa base de clientèle : «banque ouverte à tous » après la mensualisation des salariés, large diffusion des prêts aidés à la propriété (PAP) consentis pour 20 ans à des ménages à revenus modestes.

48

- Un réseau international récent. La construction d'un réseau international est engagée avec l'ouverture, en 1979 à Chicago, de la première succursale à l'étranger.

49 - L'imagination. Le Crédit Agricole se place à la pointe de l'innovation (informatique, monétique). Chaque Caisse régionale constitue, à côté de la CNCA, un laboratoire d'initiatives. De nombreuses avancées techniques ont ainsi trouvé leur origine dans une ou plusieurs Caisses régionales.

\section{Le Crédit Agricole en quelques chiffres}

50 Aujourd'hui classé parmi les premières banques mondiales, et en tout cas première banque européenne, le Crédit Agricole est une puissance financière (1 754 milliards de francs de bilan) qui dispose d'une assise commerciale forte (avec près de 15 millions de comptes, le Crédit Agricole est le banquier d'un Français sur trois) et d'un réseau de proximité sans équivalent (68 Caisses régionales et leurs 8350 agences, 5000 guichets automatiques bancaires et 10000 "points verts »). Il réunit près de 5,6 millions de sociétaires, emploie 73000 salariés et bénéficie du concours d'environ $\mathbf{4 0 0 0 0}$ administrateurs bénévoles.

\section{NOTES}

1. Directeur général de la CNCA de 1988 à 1993, Philippe Jaffré est actuellement PDG d'Elf Aquitaine. Le conseil d'administration de la CNCA a désigné pour lui succéder Lucien Douroux, Directeur général de la Caisse régionale d'île-de-France. Comme Yves Barsalou et Philippe Jaffré, il attache une grande importance à l'histoire et à la culture d'entreprise.

2. Première publication illustrée et " grand public ", cette plaquette, qui était à l'origine destinée au groupe constitué par la CNCA et ses filiales, fut diffusée aux Caisses régionales, à la demande de celles-ci.

3. Ainsi le livre Saga verte en Moselle, consacré à l'histoire de la Caisse régionale, a été distribué à l'occasion de la dernière assemblée générale de celle-ci comme Caisse autonome, avant son

Communication et organisation, $7 \mid 1995$ 
regroupement avec les Caisses de Nancy et de Bar-le-Duc. L'ouvrage a pu contribuer à faire adhérer les salariés à la politique de restructuration qu'ils voyaient mettre en œuvre.

4. Un Siècle au Présent, 192 pages. 306 illustrations, Editions Hervas, 1994. Tirage a ce jour : 30.000 exemplaires.

\section{RÉSUMÉS}

Parmi les tout premiers établissements bancaires français, le Crédit Agricole a créé une mission historique (1990). Son responsable, Christian Bosséno, administrateur financier à la Caisse nationale de Crédit Agricole, évoque la genèse de ce service spécialisé, sa raison d'être, ses objectifs, son organisation, son fonctionnement avec les Caisses régionales. Ses premières productions aussi, notamment le livre "Un Siècle au présent» publié dans le cadre de la célébration du centenaire du Crédit Agricole (1994). Instituée auprès du Secrétariat central de la Caisse nationale, la Mission historique travaille en relation étroite avec la Direction générale, la direction des Ressources humaines et la direction de la Communication. Elle a d'autre part monté un réseau de correspondants avec les Caisses régionales et les filiales. Conçue comme une unité pérenne, elle doit aussi reconstituer les archives historiques et organiser celles de demain à partir des documents d'aujourd'hui. Le récit d'une expérience originale qui a su acquérir sa pleine légitimité au sein d'un groupe à la fois uni et décentralisé.

In 1990, Crédit Agricole was one of the first French banks to set up a special service - the Mission Histoire - in order to keep alive the history of the Group. In this article. Christian Bosséno, head of the Mission and a senior officer at Caisse Nationale de Credit Agricole (CNCA), reviews the events leading up to the birth of this service as well as its organization and interaction with the Regional Banks. He discusses the service's early work, and notably the preparation of a book, " (Un Siécle au Present ». published to commemorate Credit Agricole's hundredth anniversary in 1994. Set up as part of the Central Secretariat of CNCA, the Mission Histoire works closely with the general management, the human resources department and the communications department. With the assistance of the Regional Banks and subsidiaries, it has also set up a network of correspondents. The Mission has been designed as a permanent structure and its remit further includes building up a historical archive and organizing the procedures for archiving documents for future generations. Mr Bosséno's article recounts the story of an original initiative that has gained widespread acceptance in a Group that is both decentralized and united.

\section{AUTEUR}

\section{CHRISTIAN BOSSÉNO}

Christian Bosséno est administrateur financier à la Caisse nationale de Crédit Agricole. Né en 1938, licencié en droit (économie politique) et diplômé du CESB, il est entré à la CNCA en 1963. Il a en particulier été chef de mission à l'Inspection générale puis responsable, à la direction de l'International, pendant une dizaine d'années, des relations de coopération technique avec les pays en développement. Il dirige, depuis sa création en 1990, la Mission 
Archives/Histoire de la CNCA. A ce titre, il a notamment coordonné la réalisation d'« Un siècle au présent ", livre publié à l'occasion du centenaire du Crédit Agricole. Il est par ailleurs l'auteur d'ouvrages d'analyse critique sur le cinéma et la télévision. 